\title{
Highly Accurate Biomolecular Electrostatics in Continuum Dielectric Environments
}

\author{
Y. C. ZHOU, ${ }^{1 *}$ MICHAEL FEIG, ${ }^{2,3}$ G. W. WEI ${ }^{1,4}$ \\ ${ }^{1}$ Department of Mathematics, Michigan State University, East Lansing, Michigan 48824 \\ ${ }^{2}$ Department of Chemistry, Michigan State University, East Lansing, Michigan 48824 \\ ${ }^{3}$ Department of Biochemistry and Molecular Biology, 4223 Biomedical Physical Sciences Building, \\ Michigan State University, East Lansing, Michigan 48824 \\ ${ }^{4}$ Department of Electrical and Computer Engineering, Michigan State University, East Lansing, \\ Michigan 48824 \\ Received 11 February 2007; Accepted 7 April 2007 \\ DOI 10.1002/jcc.20769 \\ Published online 16 May 2007 in Wiley InterScience (www.interscience.wiley.com).
}

\begin{abstract}
Implicit solvent models based on the Poisson-Boltzmann (PB) equation are frequently used to describe the interactions of a biomolecule with its dielectric continuum environment. A novel, highly accurate Poisson-Boltzmann solver is developed based on the matched interface and boundary (MIB) method, which rigorously enforces the continuity conditions of both the electrostatic potential and its flux at the molecular surface. The MIB based PB solver attains much better convergence rates as a function of mesh size compared to conventional finite difference and finite element based PB solvers. Consequently, highly accurate electrostatic potentials and solvation energies are obtained at coarse mesh sizes. In the context of biomolecular electrostatic calculations it is demonstrated that the MIB method generates substantially more accurate solutions of the PB equation than other established methods, thus providing a new level of reference values for such models. Initial results also indicate that the MIB method can significantly improve the quality of electrostatic surface potentials of biomolecules that are frequently used in the study of biomolecular interactions based on experimental structures.
\end{abstract}

(C) 2007 Wiley Periodicals, Inc. J Comput Chem 29: 87-97, 2008

Key words: Poisson-Boltzmann; interface flux continuity conditions; matched interference and boundary

\section{Introduction}

Continuum dielectric theory has wide applications in chemistry, physics, material science and biology such as studies of colloid solutions, ${ }^{1,2}$ membranes,${ }^{3}$ nanotubes, ${ }^{4}$ and photo-induced charge transfer. ${ }^{5}$ In structural biology, continuum dielectric theory allows a macroscopic description of the solvent in the context of implicit solvent models in which the electrostatic interactions between biomolecules and the solvent are represented by a dielectric continuum via a mean-field average over the solvent degrees of freedom. ${ }^{6,7}$ The main advantage of using implicit solvent models is a substantial reduction in computational cost over explicit representations of the solvent because of smaller system sizes for a given molecular conformation. Continuum dielectric models have been widely employed in structural biology to examine electrostatic interactions within and between biological molecules and to facilitate binding and conformational free energy estimates. ${ }^{8-17}$ They are also used increasingly to accelerate molecular dynamics simulations of biomolecules in a variety of contexts. ${ }^{18-24}$

The electrostatic potential induced by a set of explicit charges within a dielectric continuum environment is described rigorously by the Poisson equation or the Poisson-Boltzmann (PB) equation where the latter accounts for the presence of ions satisfying the Boltzmann distribution in the continuum. Solving the PB equation throughout space is not trivial, but a variety of methods have been established in the past. In particular, finite difference methods, ${ }^{10,25-27}$ finite element methods, ${ }^{8,28}$ and boundary element methods ${ }^{13,29,30}$ have been proposed and are widely used. A recent comparison of current $\mathrm{PB}$ solvers along with implementations of the Generalized Born (GB) approximation found that estimates of electrostatic solvation energies may vary by $0.25 \%$ to $0.5 \%$ with respect to the total solvation energies between different methods, ${ }^{31}$ suggesting that none of the available methods is able to reach fully

\footnotetext{
*Present address: Department of Mathematics, University of California, San Diego, La Jolla, CA 92093-0112, USA

Correspondence to: G. W. Wei; e-mail: wei@math.msu.edu

Contract/grant sponsor: NSF; contract/grant numbers: DMS-0616704, 115-0430987, 0447799

Contract/grant sponsor: Alfred P. Sloan Fellowship
} 
converged solutions. The possibility for significant uncertainties raises concerns in the practical use of $\mathrm{PB}$ theory with existing $\mathrm{PB}$ solvers. $^{32}$

Attaining highly accurate solutions of the PB equation for biological systems is hindered in part by the discontinuity of the dielectric constant across the dielectric interface ${ }^{33}$ as well as the irregularity of the interface. Mathematically, the electrostatic potential becomes non-differentiable at the interface because of the dielectric discontinuity, and this results in low order convergence properties of standard finite difference or finite element based PB solvers. ${ }^{34}$ The convergence of a numerical method can be improved by enforcing the continuities of both electrostatic potential $\phi$ and its flux at the dielectric interface, i.e., $\phi^{+}=\phi^{-}$and $\epsilon^{+} \phi_{\xi}^{+}=\epsilon^{-} \phi_{\xi}^{-}$. Here, the superscript "-" denotes the potential inside an explicitly modeled molecule and "+" represents the solvent region modeled as a continuum. The partial derivative is defined in the normal direction at the dielectric interface and $\xi$ is the unit outer normal vector. Most of the established PB solvers consider only the continuity of the electrostatic potential but neglect the continuity of the potential flux, and consequently suffer from slow convergence to an accurate solution. Highly accurate, converged solutions are in principle attainable with conventional PB solvers either by using a very fine mesh, for example with hierarchical ${ }^{35}$ or parallel $^{28}$ focusing techniques, or by refining the mesh locally based on a priori or a posterior error estimation. ${ }^{36}$ However, the large system sizes in biological applications limit the grid resolution that can be managed practically.

In a broader perspective, the PB equation represents a special type of physical and mechanical problems, which are distinguished by discontinuous coefficients across the interface and/or nondifferentiable solutions. These problems include the propagation of electromagnetic waves in heterogeneous media, the crystal growth from the liquid phase and two-phase flows. The failure of most traditional numerical methods motivated the development of a number of algorithms, such as the immersed boundary method, ${ }^{37}$ immersed interface method, ${ }^{38}$ and ghost fluid method. ${ }^{39}$ We have recently introduced the matched interface and boundary (MIB) $)^{40,41}$ method to address such problems.

In this paper we show that conventional PB solvers indeed face limitations in reaching convergent solutions, especially in the vicinity of the charges and the dielectric interface, that affect the accuracy in the application of continuum dielectric electrostatics to complex problems. An MIB method introduced here is one that is able to overcome these limitations and arrive at convergent, highly accurate electrostatic potentials with moderate computational costs. In the following, we will first describe the the theoretical underpinnings of this new method. The results of applying this method in a variety of contexts from simple electrostatic problems to biomolecular systems are then presented and compared with existing methods.

\section{Theory}

To illustrate the concept of the MIB method, we describe a onedimensional (1D) interface algorithm for the PB equation. A brief description of the 3D MIB scheme is provided. Some details of the 3D theory can be found in the Appendix.

\section{One-Dimensional Illustration}

In the MIB method, the continuity conditions of the electrostatic potential and its flux are implicitly enforced by modifying the discretization schemes near the dielectric interface. For simplicity we consider a one-dimensional (1D) case with a uniform mesh of grid spacing $h$. Let the interface be located at $x=a$ where $x_{i} \leq a \leq x_{i+1}$ for some $i$ (see Fig. 1) and assume that the dielectric constant is $\epsilon^{-}$and $\epsilon^{+}$in the left and right subdomains, respectively.

For the 1D Poisson eq. (1) with interface conditions (2)

$$
\begin{aligned}
-\left(\epsilon \phi_{x}\right)_{x} & =f(x), \\
\phi^{+}(a) & =\phi^{-}(a), \epsilon^{+} \phi_{x}^{+}(a)=\epsilon^{-} \phi_{x}^{-}(a) .
\end{aligned}
$$

A straightforward application of the finite difference scheme at $x_{i}$ gives

$$
-\frac{\epsilon_{i+1 / 2} \phi_{i+1}-\left(\epsilon_{i+1 / 2}+\epsilon_{i-1 / 2}\right) \phi_{i}+\epsilon_{i-1 / 2} \phi_{i-1}}{h^{2}}=f\left(x_{i}\right),
$$

where $\epsilon_{i+1 / 2}$ and $\epsilon_{i-1 / 2}$ are the values of dielectric constant at $x_{i+1 / 2}$ and $x_{i-1 / 2}$, respectively. This commonly employed discretization delivers a second-order convergence only if both $\phi(x)$ and $\epsilon(x)$ are continuous. In order to restore this high order convergence, the MIB method modifies the standard finite difference scheme by first smoothly extending the subdomains near the interface, and then applying the standard finite difference scheme on the extended subdomains.

As seen from Figure 1, a second-order central difference scheme at $x_{i}$ involves grid points $x_{i-1}, x_{i}$ and $x_{i+1}$, where $x_{i+1}$ is located on the other side of the interface. In the left subdomain, the finite difference approximation to eq. (1) that involves the grid point on the other domain is the one at $x_{i}$; thus it is sufficient to extend the left subdomain up to $x_{i+1}$ to accommodate the discretization at $x_{i}$. Similarly, the right subdomain would be left-extended up to $x_{i}$. The solution values on these two extended domains are referred to as fictitious values $f(x)$ to distinguish them from the real solution values $\phi(x)$ at the same locations. These two fictitious values $f_{i}$ and $f_{i+1}$ are solved from the approximation of interface conditions (2), and are expressed in terms of $\phi_{i-1}, \phi_{i}, \phi_{i+1}$, and $\phi_{i+2}$. The discretization of eq. (1) with

$$
-\frac{\epsilon_{i+1 / 2} f_{i+1}-\left(\epsilon_{i+1 / 2}+\epsilon_{i-1 / 2}\right) \phi_{i}+\epsilon_{i-1 / 2} \phi_{i-1}}{h^{2}}=f\left(x_{i}\right)
$$

is therefore conducted on a smooth domain and able to preserve the interface conditions (2), thus enabling the numerical approach to achieve high order convergence.

\section{Interface Treatment in Three Dimensions}

For the $\mathrm{PB}$ equation in a 3D space with an irregular dielectric interface, we also have two interface conditions anywhere along the interface 


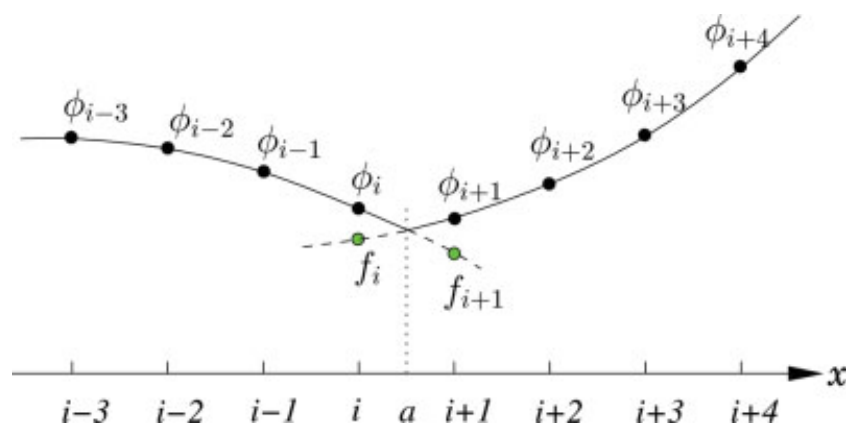

Figure 1. Illustration of fictitious values for a $1 \mathrm{D}$ problem. [Color figure can be viewed in the online issue, which is available at www. interscience.wiley.com.]

$$
\begin{gathered}
{[\phi]=\phi^{+}-\phi^{-}=0} \\
{\left[\epsilon \phi_{\xi}\right]=\epsilon^{+} \phi_{\xi}^{+}-\epsilon^{-} \phi_{\xi}^{-} .}
\end{gathered}
$$

However, the continuity of the potential flux (6) is now defined in the normal direction $\xi$, which is in general not aligned with any coordinate direction. Since we solve the PB equation on the Cartesian mesh, we are particularly interested in implementing these interface conditions on each intersecting point of the interface and the mesh. At a given intersecting point, it is convenient to introduce a coordinate transform from $(\xi, \eta, \zeta)$ to $(x, y, z)$, where $\eta$ and $\zeta$ are the tangential and binormal directions, respectively. We therefore locally project the flux continuity condition into the Cartesian mesh

$$
\begin{aligned}
{\left[\epsilon \phi_{\xi}\right]=} & \epsilon^{+}\left(\cos \psi \cos \theta \phi_{x}^{+}+\cos \psi \sin \theta \phi_{y}^{+}+\sin \psi \phi_{z}^{+}\right) \\
& -\epsilon^{-}\left(\cos \psi \cos \theta \phi_{x}^{-}+\cos \psi \sin \theta \phi_{y}^{-}+\sin \psi \phi_{z}^{-}\right),
\end{aligned}
$$

where $\psi$ is the angle between $\xi$ and its projection on $x-y$ plane while $\theta$ is the angle between this projection and $x$-direction. Two additional interface conditions can be obtained by differentiating the continuity condition (5) of the electrostatic potential with respect to the tangential direction $\eta$

$$
\phi_{\eta}^{+}-\phi_{\eta}^{-}=\left(-\sin \theta \phi_{x}^{+}+\cos \theta \phi_{y}^{+}\right)-\left(-\sin \theta \phi_{x}^{-}+\cos \theta \phi_{y}^{-}\right)
$$

and the binormal direction $\zeta$

$$
\begin{aligned}
\phi_{\zeta}^{+}-\phi_{\zeta}^{-} & =\left(-\sin \psi \cos \theta \phi_{x}^{+}-\sin \psi \sin \theta \phi_{y}^{+}+\cos \psi \phi_{z}^{+}\right) \\
& -\left(-\sin \psi \cos \theta \phi_{x}^{-}-\sin \psi \sin \theta \phi_{y}^{-}+\cos \psi \phi_{z}^{-}\right)
\end{aligned}
$$

Obviously, one can keep creating higher-order interface conditions by further differentiating these three first-order interface conditions. However, further differentiation will generate higher-order partial derivatives, which are numerically unfavorable. In our MIB method, we limit ourselves to these four interface conditions. One of the difficulties in enforcing these interface conditions is the calculation of the partial derivatives at each intersecting point. To simplify the problem, we will eliminate two partial derivatives most difficult to calculate from three first order interface conditions, eqs. (7)-(9). Figure 2 illustrates a case where the interface intersects the $x$-mesh line at point $\left(x_{0}, y_{0}, z_{0}\right)$. Obviously, it is more difficult to compute the partial derivatives in the $y$ - and $z$-directions at $\left(x_{0}, y_{0}, z_{0}\right)$. We therefore locally regard the $x$-direction as the primary coordinate direction, and eliminate two partial derivatives from two other directions. For example, to eliminate $\phi_{y}^{-}$and $\phi_{z}^{-}$from eqs. (7)-(9), we have

$$
\begin{aligned}
{\left[\epsilon \phi_{\xi}\right]-\epsilon^{-} \tan \psi\left[\phi_{\zeta}\right] } & +C_{\eta}\left[\phi_{\eta}\right] \\
& =C_{x}^{+} \phi_{x}^{+}+C_{x}^{-} \phi_{x}^{-}+C_{y}^{+} \phi_{y}^{+}+C_{z}^{+} \phi_{z}^{+},
\end{aligned}
$$

where

$$
\begin{aligned}
C_{\eta}= & -\epsilon^{-} \tan \theta / \cos \psi, \\
C_{x}^{+}= & \epsilon^{+} \cos \psi \cos \theta+\epsilon^{-} \tan \psi \sin \psi \cos \theta \\
& +\epsilon^{-} \sin \theta \tan \theta / \cos \psi, \\
C_{x}^{-}= & -\epsilon^{-} /(\cos \psi \cos \theta), \\
C_{y}^{+}= & \left(\epsilon^{+}-\epsilon^{-}\right) \cos \psi \sin \theta, \\
C_{z}^{+}= & \left(\epsilon^{+}-\epsilon^{-}\right) \sin \psi .
\end{aligned}
$$

It turns out that there are 12 possible combinations to select two partial derivatives from six of them. A complete description of these combinations is given in the Appendix. At each intersecting point, only one of these 12 combinations will be selected according to the local topology. This interface flux condition is combined with the continuity condition (5) to determine two fictitious values. In particular, one of the first four flux conditions, eqs. (A1)-(A4) in the

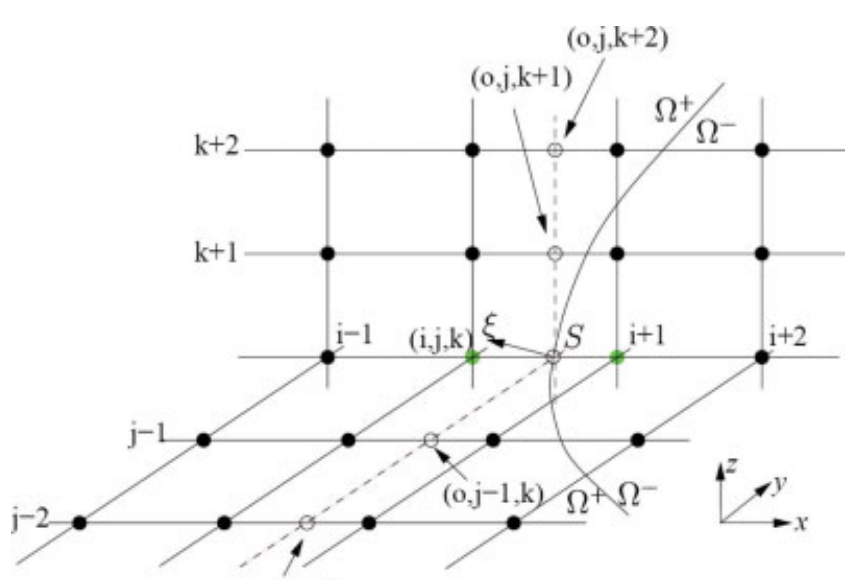

$(0, j-2, k)$

Figure 2. Local topology around irregular point $(i, j, k)$. The interface crosses the $x$-mesh line at the point $S=\left(x_{0}, y_{0}, z_{0}\right)$ between $(i, j, k)$ and $(i+1, j, k)$, which are two irregular points, on which two fictitious values, $f_{i, j, k}$ and $f_{i+1, j, k}$ are defined (marked with green dots). Two auxiliary lines (dashed line) are sketched passing through $S$, one on the $x-y$ plane and the other on the $x-z$ plane. Two auxiliary points (in empty circle) $(0, j, k+1)$ and $(0, j, k+2)$ are placed on the auxiliary line on the $x-z$ plane to facilitate the discretization of $\phi_{z}^{+}$; also, two auxiliary points $(0, j, k+1)$ and $(0, j, k+2)$ are placed on the auxiliary line on the $x-y$ plane to facilitate the discretization of $\phi_{y}^{+}$. [Color figure can be viewed in the online issue, which is available at www.interscience.wiley.com.] 
Appendix, will be chosen to solve for a pair of fictitious values in $x$-direction. To solve for fictitious values in the $y$ - or $z$-direction, one can select one of eqs. (A5)-(A8) or eqs. (A9)-(A12), respectively. In the rest of this section, we describe the determination of two fictitious values in Fig. 2 by using interface conditions (5) and (10). The determination of fictitious values with other sets of conditions can be accomplished similarly.

In Fig. 2, the dielectric interface intersects the $x$-mesh line at point $S$ between $(i, j, k)$ and $(i+1, j, k)$. These two grid points are the irregular points. The solution of interior domain will be extended to point $(i+1, j, k)$ as $f(i+1, j, k)$, whereas the exterior domain will be continued to point $(i, j, k)$ to define $f(i, j, k)$. With these two fictitious values the interface condition (5) can be approximated as

$$
\begin{aligned}
& \left(w_{0, i}^{+} f_{i, j, k}+w_{0, i+1}^{+} \phi_{i+1, j, k}+w_{0, i+2}^{+} \phi_{i+2, j, k}\right) \\
& \quad-\left(w_{0, i-1}^{-} \phi_{i-1, j, k}+w_{0, i}^{-} \phi_{i, j, k}+w_{0, i+1}^{-} f_{i+1, j, k}\right)=[\phi],
\end{aligned}
$$

where $w$ are the finite difference weights. Their first subscript can be either 0 or 1 , labeling the interpolation and first order derivative, respectively. The second subscript labels the position. The superscript can be either + or - , labeling the + side or the side of the interface. In a similar way we can also obtain the finite difference approximation of partial derivatives $\phi_{x}^{-}$and $\phi_{x}^{+}$ in eq. (10). The approximation of other two derivatives $\phi_{y}^{+}$and $\phi_{z}^{+}$ at point $\left(x_{0}, y_{0}, z_{0}\right)$, however, remains a problem because $\left(x_{0}, y_{0}, z_{0}\right)$ is located neither on a $y$-mesh line nor on a $z$-mesh line. To overcome this difficulty, we add two auxiliary lines passing $\left(x_{0}, y_{0}, z_{0}\right)$, one in $x-y$ plane and the other in $x-z$ plane, and choose two auxiliary points on each auxiliary line to support one-sided finite difference schemes for $\phi_{y}^{+}$and $\phi_{z}^{+}$. This makes it possible to approximate eq. (10) as

$$
\begin{aligned}
{\left[\epsilon \phi_{\xi}\right]-\epsilon^{-} } & \tan \psi\left[\phi_{\zeta}\right]+C_{\eta}\left[\phi_{\eta}\right] \\
& =C_{x}^{+}\left(w_{1, i}^{+} f_{i, j, k}+w_{1, i+1}^{+} \phi_{i+1, j, k}+w_{1, i+2}^{+} \phi_{i+2, j, k}\right) \\
& +C_{x}^{-}\left(w_{1, i-1}^{-} \phi_{i-1, j, k}+w_{1, i}^{-} \phi_{i, j, k}+w_{1, i+1}^{-} f_{i+1, j, k}\right) \\
& +C_{y}^{+}\left(p_{0, j} \phi_{0, j, k}^{+}+p_{0, j-1} \phi_{0, j-1, k}^{+}+p_{0, j-2} \phi_{0, j-2, k}^{+}\right) \\
& +C_{z}^{+}\left(q_{0, k} \phi_{0, j, k}^{+}+q_{0, k+1} \phi_{0, j, k+1}^{+}+q_{0, k+2} \phi_{0, j, k+2}^{+}\right)
\end{aligned}
$$

where $p_{0,:}$ is the finite difference weights for $\phi_{y}^{+}$at auxiliary nodes $(0, j, k),(0, j-1, k),(0, j-2, k)$, and $q_{0,:}$ is the finite difference weights for $\phi_{z}^{+}$at auxiliary nodes $(0, j, k),(0, j, k+1),(0, j, k+2)$.

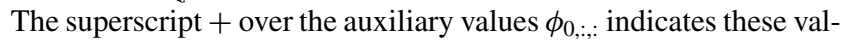
ues are on the + side of the interface. Fictitious values $f_{i, j, k}$ and $f_{i+1, j, k}$ can be solved from eqs. (11) and (12) in terms of solution values at four normal grid nodes, $\phi_{i-1, j, k}, \phi_{i, j, k}, \phi_{i+1, j, k}, \phi_{i+2, j, k}$, five auxiliary points, $\phi_{0, j, k}^{+}, \phi_{0, j-1, k}^{+}, \phi_{0, j-2, k}^{+}, \phi_{0, j, k+1}^{+}, \phi_{0, j, k+2}^{+}$, and four given jumps $[\phi],\left[\epsilon \phi_{\xi}\right],\left[\phi_{\eta}\right],\left[\phi_{\zeta}\right]$ :

$$
\begin{gathered}
f_{i, j, k}=C_{1}^{i} \phi_{i-1, j, k}+C_{2}^{i} \phi_{i, j, k}+C_{3}^{i} \phi_{i+1, j, k}+C_{4}^{i} \phi_{i+2, j, k}+C_{5}^{i} \phi_{0, j, k}^{+} \\
+C_{6}^{i} \phi_{0, j-1, k}^{+}+C_{7}^{i} \phi_{0, j-2, k}^{+}+C_{8}^{i} \phi_{0, j, k}^{+}+C_{9}^{i} \phi_{0, j, k+1}^{+} \\
+C_{10}^{i} \phi_{0, j, k+2}^{+}+C_{11}^{i}[\phi]+C_{12}^{i}\left[\epsilon \phi_{\xi}\right]+C_{13}^{i}\left[\phi_{\eta}\right]+C_{14}^{i}\left[\phi_{\zeta}\right],
\end{gathered}
$$

$$
\begin{aligned}
f_{i+1, j, k}=C_{1}^{i+1} \phi_{i-1, j, k}+C_{2}^{i+1} \phi_{i, j, k}+C_{3}^{i+1} \phi_{i+1, j, k} \\
+C_{4}^{i+1} \phi_{i+2, j, k}+C_{5}^{i+1} \phi_{0, j, k}^{+}+C_{6}^{i+1} \phi_{0, j-1, k}^{+}+C_{7}^{i+1} \phi_{0, j-2, k}^{+} \\
\quad+C_{8}^{i+1} \phi_{0, j, k}^{+}+C_{9}^{i+1} \phi_{0, j, k+1}^{+}+C_{10}^{i+1} \phi_{0, j, k+2}^{+} \\
\quad+C_{11}^{i+1}[\phi]+C_{12}^{i+1}\left[\epsilon \phi_{\xi}\right]+C_{13}^{i+1}\left[\phi_{\eta}\right]+C_{14}^{i+1}\left[\phi_{\zeta}\right],
\end{aligned}
$$

where coefficients $C$ can be easily determined from eqs. (11) and (12). However, in these two expressions of fictitious values, the solution values at auxiliary points have not been defined yet. In the MIB, these values are obtained via the interpolation of the available normal grid values from the same side of the interface. For example, $\phi_{0, j-1, k}^{+}$can be interpolated from potential values on $(i-1, j-1, k),(i, j-1, k),(i+1, j-1, k)$, and $\phi_{0, j-2, k}^{+}$is interpolated from potential values on $(i-1, j-2, k),(i, j-2, k),(i+1, j-2, k)$. Similarly, auxiliary values $\phi_{0, j, k-1}^{+}$and $\phi_{0, j, k-2}^{+}$are interpolated from $(i-2, j, k+1),(i-1, j, k+1),(i, j, k+1)$ and $(i-1, j, k+$ $2),(i, j, k+2),(i+1, j, k+2)$, respectively. It is also noted that the first interface condition, eq. (11), should be used to compute the auxiliary value $\phi_{0, j, k}^{+}$as $\left(w_{0, i}^{+} f_{i, j, k}+w_{0, i+1}^{+} \phi_{i+1, j, k}+w_{0, i+2}^{+} \phi_{i+2, j, k}\right)$ or $\left(w_{0, i-1}^{-} \phi_{i-1, j, k}+w_{0, i}^{-} \phi_{i, j, k}+w_{0, i+1}^{-} f_{i+1, j, k}\right)+[\phi]$. By distributing the expansion coefficients of the fictitious values on these auxiliary points to their respective interpolation node values, we can obtain the final expressions of the fictitious values $f_{i, j, k}$ and $f_{i+1, j, k}$. Specifically, we have

$$
\begin{aligned}
\phi_{0, j, k}^{+} & =\left(w_{0, i-1}^{-} \phi_{i-1, j, k}+w_{0, i}^{-} \phi_{i, j, k}+w_{0, i+1}^{-} f_{i+1, j, k}\right)+[\phi], \\
\phi_{0, j-1, k}^{+} & =\mathbf{I}_{j-1} \cdot\left(\phi_{i-1, j-1, k}, \phi_{i, j-1, k}, \phi_{i+1, j-1, k}\right)^{T}, \\
\phi_{0, j-2, k}^{+} & =\mathbf{I}_{j-2} \cdot\left(\phi_{i-1, j-2, k}, \phi_{i, j-2, k}, \phi_{i+1, j-2, k}\right)^{T}, \\
\phi_{0, j, k+1}^{+} & =\mathbf{I}_{k+1} \cdot\left(\phi_{i-2, j, k+1}, \phi_{i-1, j, k+1}, \phi_{i, j, k+1}\right)^{T}, \\
\phi_{0, j, k+2}^{+} & =\mathbf{I}_{k+2} \cdot\left(\phi_{i-1, j, k+2}, \phi_{i, j, k+2}, \phi_{i+1, j, k+2}\right)^{T},
\end{aligned}
$$

where $\mathbf{I}$ is the vector consisting of corresponding interpolation weights. Then we obtain the following expressions for

$$
\begin{aligned}
f_{i, j, k}=C_{1}^{i} \phi_{i-1, j, k}+C_{2}^{i} \phi_{i, j, k}+C_{3}^{i} \phi_{i+1, j, k}+C_{4}^{i} \phi_{i+2, j, k}+\left(C_{5}^{i}+C_{8}^{i}\right) \\
\times\left(w_{0, i-1}^{-} \phi_{i-1, j, k}+w_{0, i}^{-} \phi_{i, j, k}+w_{0, i+1}^{-} f_{i+1, j, k}+[\phi]\right) \\
\quad+C_{6}^{i} \cdot \mathbf{I}_{j-1} \cdot\left(\phi_{i-1, j-1, k}, \phi_{i, j-1, k}, \phi_{i+1, j-1, k}\right)^{T} \\
+C_{7}^{i} \cdot \mathbf{I}_{j-2} \cdot\left(\phi_{i-1, j-2, k}, \phi_{i, j-2, k}, \phi_{i+1, j-2, k}\right)^{T} \\
+C_{9}^{i} \cdot \mathbf{I}_{k+1} \cdot\left(\phi_{i-2, j, k+1}, \phi_{i-1, j, k+1}, \phi_{i, j, k+1}\right)^{T} \\
+C_{10}^{i} \cdot \mathbf{I}_{k+2} \cdot\left(\phi_{i-1, j, k+2}, \phi_{i, j, k+2}, \phi_{i+1, j, k+2}\right)^{T} \\
\quad+C_{11}^{i}[\phi]+C_{12}^{i}\left[\epsilon \phi_{\xi}\right]+C_{13}^{i}\left[\phi_{\eta}\right]+C_{14}^{i}\left[\phi_{\zeta}\right],
\end{aligned}
$$

and

$$
\begin{aligned}
& f_{i+1, j, k}=C_{1}^{i+1} \phi_{i-1, j, k}+C_{2}^{i+1} \phi_{i, j, k}+C_{3}^{i+1} \phi_{i+1, j, k}+C_{4}^{i+1} \phi_{i+2, j, k} \\
& \quad+\left(C_{5}^{i+1}+C_{8}^{i+1}\right)\left(w_{0, i-1}^{-} \phi_{i-1, j, k}+w_{0, i}^{-} \phi_{i, j, k}+w_{0, i+1}^{-} f_{i+1, j, k}+[\phi]\right)
\end{aligned}
$$




$$
\begin{aligned}
& +C_{6}^{i+1} \cdot \mathbf{I}_{j-1} \cdot\left(\phi_{i-1, j-1, k}, \phi_{i, j-1, k}, \phi_{i+1, j-1, k}\right)^{T} \\
& +C_{7}^{i+1} \cdot \mathbf{I}_{j-2} \cdot\left(\phi_{i-1, j-2, k}, \phi_{i, j-2, k}, \phi_{i+1, j-2, k}\right)^{T} \\
& +C_{9}^{i+1} \cdot \mathbf{I}_{k+1} \cdot\left(\phi_{i-2, j, k+1}, \phi_{i-1, j, k+1}, \phi_{i, j, k+1}\right)^{T} \\
& +C_{10}^{i+1} \cdot \mathbf{I}_{k+2} \cdot\left(\phi_{i-1, j, k+2}, \phi_{i, j, k+2}, \phi_{i+1, j, k+2}\right)^{T} \\
& +C_{11}^{i+1}[\phi]+C_{12}^{i+1}\left[\epsilon \phi_{\xi}\right]+C_{13}^{i+1}\left[\phi_{\eta}\right]+C_{14}^{i+1}\left[\phi_{\zeta}\right] .
\end{aligned}
$$

This completes the determination of fictitious values $f_{i, j, k}$ and $f_{i+1, j, k}$ in the $x$-direction. By means of fictitious values, we can discretize the PB equation by using the standard finite difference scheme. For example, $\frac{\partial^{2} \phi}{\partial x^{2}}$ at the points $(i, j, k)$ and $(i+1, j, k)$ are modified accordingly by incorporating these fictitious values

$$
\begin{array}{r}
\left.\frac{\partial^{2} \phi}{\partial x^{2}}\right|_{i, j, k}=\frac{f_{i+1, j, k}-2 \phi_{i, j, k}+\phi_{i-1, j, k}}{h^{2}} \text { at }(i, j, k), \\
\left.\frac{\partial^{2} \phi}{\partial x^{2}}\right|_{i+1, j, k}=\frac{\phi_{i+2, j, k}-2 \phi_{i+1, j, k}+f_{i-1, j, k}}{h^{2}} \text { at }(i+1, j, k),
\end{array}
$$

and replacing $f_{i, j, k}$ and $f_{i+1, j, k}$ with their respective representations in Eq. (15) or Eq. (16).

Although these fictitious values $f_{i, j, k}$ or $f_{i+1, j, k}$ are solved in $x$-direction, they represent the smooth continuation of the solution on the respective subdomain to the point they are located. Therefore they can be used for the formulation of difference schemes for any other partial derivatives involving these grid points. This approach is particularly valuable when the geometry is very complex at some grid locations where the determination of fictitious values along some directions becomes a problem. It is seen from this procedure that the MIB method reduces the solution of fictitious values in a 3D problem into a 1D-like one. The reader is also referred to Refs. 40-43 for more detailed mathematical considerations and applications of the MIB method.

\section{Results}

In all test cases, the dielectric constant is taken as $\epsilon^{-}=1$ and $\epsilon^{+}=80$. The probe radius is set to $1.4 \AA$ when it is applicable. Electrostatic potential is solved with the MIB method and for comparison also with $\mathrm{PBEQ},{ }^{44}$ a representative finite difference $\mathrm{PB}$ solver from CHARMM,${ }^{45}$ and APBS, ${ }^{46-49}$ a multigrid PB solver recently developed primarily for massively parallel computing. The APBS has both finite difference and finite element formulations, while its finite difference function is used the present work. From the electrostatic potentials in vacuum, $\phi_{\text {vac }}$ and in the presence of the dielectric environment, $\phi_{\text {dielec }}$, the electrostatic free energy of solvation, $\Delta G_{\text {solv,elec }}$ is calculated from the explicit charges $q_{i}$ at positions $r_{i}$ as:

$$
\Delta G_{\text {solv,elec }}=\frac{1}{2} \sum_{i} q_{i}\left[\phi_{\text {dielec }}\left(r_{i}\right)-\phi_{\text {vac }}\left(r_{i}\right)\right] .
$$

\section{Analytical Test Cases}

The PB equation admits analytical solutions only for a limited number of simple cases. In order to establish the validity and performance of the MIB method we first consider centered and off-centered unit charges within a spherical dielectric boundary, for which exact solutions are available due to Kirkwood. ${ }^{50}$

\section{Centered Unit Charge}

Table 1 lists the results for the case of a centered unit charge within a sphere. It is found that a convergent solvation energy can be obtained with all PB solvers. However, the MIB method achieves near-exact results already with a grid spacing of $0.5 \AA$ while a grid spacing of $0.05 \AA$ is necessary for the standard finite difference and finite element solvers to reach values close to the analytical solutions. The most significant difference occurs in the accuracy of the electrostatic potential near the surface at the mesh size of $0.5 \AA$; at this resolution, the surface potentials from the PBEQ and the APBS solvers deviate from the exact value on average by $23 \%$ with a maximum of $84 \%$. Although these errors are reduced with decreasing grid spacing their orders of magnitude are larger than those of the MIB method, with more accurate surface potentials are already obtained at a grid spacing of $0.5 \AA$. Furthermore, the solution becomes essentially exact when a grid spacing of $0.05 \AA$ is employed.

Table 1. Electrostatic Solvation Energy $\Delta G$ in $\mathrm{kcal} / \mathrm{mol}$ and the Error in the Surface Potential for a Spherical Dielectric System (Radius: $2 \AA, \epsilon^{-}=1$, $\epsilon^{+}=80$ ) with a Centered Unit Charge as a Function of Grid Spacing $h^{\mathrm{a}}{ }^{\mathrm{a}}$

\begin{tabular}{ccccc}
\hline$h(\AA)$ & $\Delta G$ & $E_{1}$ & $E_{2}$ & $E_{3}$ \\
\hline MIB & & & & \\
0.50 & -81.97 & 2.63 & 8.54 & 1.65 \\
0.20 & -81.98 & 0.30 & 1.55 & 0.36 \\
0.10 & -81.98 & 0.04 & 0.37 & 0.09 \\
0.05 & -81.98 & 0.01 & 0.13 & 0.01 \\
PBEQ & & & & \\
0.50 & -85.78 & 17.05 & 84.26 & 23.31 \\
0.20 & -82.84 & 7.51 & 74.44 & 14.08 \\
0.10 & -82.49 & 3.84 & 62.30 & 12.52 \\
0.05 & -82.20 & 1.94 & 46.95 & 8.49 \\
APBS & & & & \\
0.50 & -85.85 & 17.06 & 84.26 & 23.31 \\
0.20 & -82.58 & 7.50 & 74.43 & 12.54 \\
0.10 & -82.27 & 3.83 & 62.30 & 10.55 \\
0.05 & -82.03 & 1.89 & 46.18 & 6.38 \\
\hline
\end{tabular}

${ }^{\mathrm{a}}$ The exact solvation energy from the analytical solution is $-81.98 \mathrm{kcal} / \mathrm{mol}$. $E_{1}=\max |\phi(x, y, z)-\tilde{\phi}(x, y, z)|$ is the maximum absolute error in the electrostatic potential. $E_{2}=100 \times \max \left|\frac{\phi(x, y, z)-\tilde{\phi}(x, y, z)}{\phi(x, y, z)}\right|$ is the maximum percentage (relative) error in the electrostatic potential. $E_{3}=100 \times$ $\sum_{N_{i r r}}\left|\frac{\phi(x, y, z)-\tilde{\phi}(x, y, z)}{\phi(x, y, z)}\right| / N_{\text {irr }}$ is the average percentage (relative) error in the electrostatic potential near the molecular surface. Here, $\tilde{\phi}(x, y, z)$ and $\phi(x, y, z)$ are the computed and exact electrostatic potential, respectively. $N_{\text {irr }}$ is the total number of irregular grid points where the modified difference scheme is used. 
The dramatic improvements of the MIB solver over conventional finite difference and finite element solvers reflect different convergence properties as a function of grid spacing. The MIB solver has a convergence rate of 2 , which is indicated by a fourfold decrease in all three errors as the grid spacing is halved. Such a uniform convergence is attributed to the implicit enforcement of the flux jump condition at the dielectric interface. In contrast, the PBEQ and the APBS have similarly low convergence rates of around 0.3 .

\section{Off-Center Charges Inside Sphere}

A slightly more complicated test case where analytical solutions are still available involves two off-center charges within a spherical dielectric region. This case tests the accuracy of the MIB method as interacting charges approach the dielectric interface. Results are given in Table 2 for the MIB solver and the finite difference PBEQ solver. APBS results are not shown as they are generally similar to those from PBEQ. For off-center distances of the charges between $0.2 \AA$ and about $1.0 \AA$ the MIB method also approximates the exact solution much more closely and at coarser grid spacings than the PBEQ solver. However, as the charges approach the dielectric interface closer $(a \geq 1.2)$ the error in the surface potential increases noticeably with the MIB method and consequently estimates of the solvation energy become less accurate. Indeed, for charges at an offcenter distance of $1.5 \AA$ (or $0.5 \AA$ from the surface) the solvation energies obtained with the MIB method are similar to the standard finite difference results. This degradation of performance with the interface method as charges approach the dielectric surface closer than 1-2 grid points is a result of the mixing between grid points carrying the interface information and the grid points carrying the singular charges. As Table 2 shows, the performance of the standard finite difference solver is largely uniform irrespective of where the charges are located with respect to the dielectric boundary. However, in practical biomolecular applications partial charges at atomic sites of a given biomolecule are typically at least $1 \AA$ from the dielectric surface depending on the parameters of a given biomolecular force field.

\section{Molecular Surface Test Case}

The dielectric boundary of biomolecules is typically defined as the molecular surface ${ }^{33}$ that takes into account the solventinaccessibility of crevices that are smaller than the size of a solvent molecule. The explicit enforcement of the interface conditions at the molecular surface with the MIB method necessitates an explicitly defined molecular surface such that its intersecting points with the mesh lines can be calculated to a sufficient accuracy which is consistent with the accuracy of the approximation to the PoissonBoltzmann equation. Although the analytical representation of the molecular surface is generally unavailable for a polyatomic molecule, a triangulated surface generated with the MSMS program $^{51}$ is used as an approximation of the surface and its normal directions. This information is interpolated to required MIB mesh locations. We note that molecular surface information generated

Table 2. Electrostatic Solvation Energy $\Delta G$ and Errors in the Surface Potential as Described in Table 1 for a Sphere with Two Positive Unit Charges at $(0, a, 0)$ and $(0,0, a)$ within a 2 - $\AA$ Sphere.

\begin{tabular}{|c|c|c|c|c|c|c|c|c|c|c|}
\hline \multirow{3}{*}{$\begin{array}{l}a \\
(\AA)\end{array}$} & \multirow{3}{*}{$\begin{array}{c}h \\
(\AA)\end{array}$} & & & & \multicolumn{6}{|c|}{ Errors in surface potential } \\
\hline & & \multicolumn{3}{|c|}{$\Delta G$ in $\mathrm{kcal} / \mathrm{mol}$} & \multicolumn{3}{|c|}{ MIB } & \multicolumn{3}{|c|}{ PBEQ } \\
\hline & & Exact & MIB & PBEQ & $E_{1}$ & $E_{2}$ & $E_{3}$ & $E_{1}$ & $E_{2}$ & $E_{3}$ \\
\hline 0.2 & $\begin{array}{l}0.5 \\
0.2 \\
0.1\end{array}$ & -329.56 & $\begin{array}{l}-329.55 \\
-329.51 \\
-329.54\end{array}$ & $\begin{array}{l}-345.07 \\
-333.06 \\
-331.60\end{array}$ & $\begin{array}{r}10.20 \\
0.78 \\
0.10\end{array}$ & $\begin{array}{r}12.05 \\
1.73 \\
0.42\end{array}$ & $\begin{array}{l}1.83 \\
0.36 \\
0.11\end{array}$ & $\begin{array}{r}41.46 \\
18.05 \\
9.14\end{array}$ & $\begin{array}{l}84.81 \\
75.79 \\
64.16\end{array}$ & $\begin{array}{l}24.67 \\
15.25 \\
13.02\end{array}$ \\
\hline 0.4 & $\begin{array}{l}0.5 \\
0.2 \\
0.1\end{array}$ & -334.58 & $\begin{array}{l}-334.64 \\
-334.53 \\
-334.57\end{array}$ & $\begin{array}{l}-350.85 \\
-338.25 \\
-336.72\end{array}$ & $\begin{array}{r}11.62 \\
1.01 \\
0.11\end{array}$ & $\begin{array}{r}13.60 \\
1.87 \\
0.64\end{array}$ & $\begin{array}{l}1.75 \\
0.36 \\
0.21\end{array}$ & $\begin{array}{l}49.43 \\
21.59 \\
10.94\end{array}$ & $\begin{array}{l}85.34 \\
76.98 \\
65.85\end{array}$ & $\begin{array}{l}24.56 \\
15.14 \\
12.90\end{array}$ \\
\hline 0.6 & $\begin{array}{l}0.5 \\
0.2 \\
0.1\end{array}$ & -343.38 & $\begin{array}{l}-343.94 \\
-343.37 \\
-343.38\end{array}$ & $\begin{array}{l}-360.82 \\
-347.35 \\
-345.68\end{array}$ & $\begin{array}{r}12.67 \\
1.38 \\
0.18\end{array}$ & $\begin{array}{r}21.72 \\
2.08 \\
0.51\end{array}$ & $\begin{array}{l}2.02 \\
0.36 \\
0.09\end{array}$ & $\begin{array}{l}57.34 \\
25.95 \\
13.34\end{array}$ & $\begin{array}{l}85.61 \\
78.11 \\
67.75\end{array}$ & $\begin{array}{l}24.43 \\
14.94 \\
12.71\end{array}$ \\
\hline 0.8 & $\begin{array}{l}0.5 \\
0.2 \\
0.1\end{array}$ & -356.90 & $\begin{array}{l}-358.87 \\
-356.93 \\
-356.95\end{array}$ & $\begin{array}{l}-376.24 \\
-361.35 \\
-359.47\end{array}$ & $\begin{array}{r}10.35 \\
2.12 \\
0.30\end{array}$ & $\begin{array}{r}32.56 \\
2.48 \\
0.66\end{array}$ & $\begin{array}{l}2.47 \\
0.38 \\
0.09\end{array}$ & $\begin{array}{l}64.08 \\
31.63 \\
16.87\end{array}$ & $\begin{array}{l}85.52 \\
79.24 \\
69.91\end{array}$ & $\begin{array}{l}24.13 \\
14.63 \\
12.38\end{array}$ \\
\hline 1.0 & $\begin{array}{l}0.5 \\
0.2 \\
0.1\end{array}$ & -377.35 & $\begin{array}{l}-379.09 \\
-377.49 \\
-377.38\end{array}$ & $\begin{array}{l}-397.36 \\
-382.47 \\
-380.30\end{array}$ & $\begin{array}{r}19.06 \\
3.58 \\
0.58\end{array}$ & $\begin{array}{r}34.59 \\
3.02 \\
0.94\end{array}$ & $\begin{array}{l}3.01 \\
0.44 \\
0.10\end{array}$ & $\begin{array}{l}67.92 \\
39.19 \\
22.32\end{array}$ & $\begin{array}{l}85.22 \\
80.33 \\
72.44\end{array}$ & $\begin{array}{l}23.62 \\
14.15 \\
11.89\end{array}$ \\
\hline 1.2 & $\begin{array}{l}0.5 \\
0.2 \\
0.1\end{array}$ & -409.90 & $\begin{array}{l}-426.16 \\
-410.30 \\
-409.98\end{array}$ & $\begin{array}{l}-437.68 \\
-415.66 \\
-413.34\end{array}$ & $\begin{array}{r}31.71 \\
5.80 \\
1.34\end{array}$ & $\begin{array}{r}79.56 \\
4.83 \\
1.40\end{array}$ & $\begin{array}{l}6.14 \\
0.53 \\
0.13\end{array}$ & $\begin{array}{l}66.99 \\
48.68 \\
31.00\end{array}$ & $\begin{array}{l}84.68 \\
81.33 \\
75.12\end{array}$ & $\begin{array}{l}23.07 \\
13.42 \\
11.15\end{array}$ \\
\hline 1.5 & $\begin{array}{l}0.5 \\
0.2 \\
0.1\end{array}$ & -516.18 & $\begin{array}{l}-540.35 \\
-525.41 \\
-516.80\end{array}$ & $\begin{array}{l}-541.39 \\
-526.10 \\
-519.27\end{array}$ & $\begin{array}{r}42.76 \\
24.78 \\
6.37\end{array}$ & $\begin{array}{r}84.43 \\
20.90 \\
3.81\end{array}$ & $\begin{array}{l}8.07 \\
2.02 \\
0.39\end{array}$ & $\begin{array}{l}50.03 \\
59.49 \\
51.51\end{array}$ & $\begin{array}{l}80.21 \\
81.02 \\
77.78\end{array}$ & $\begin{array}{r}21.20 \\
11.26 \\
9.20\end{array}$ \\
\hline
\end{tabular}




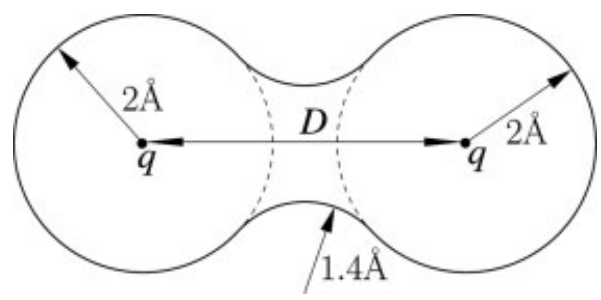

Figure 3. Two-sphere model with molecular surface. The radii of both spheres are $2 \AA$. A unit positive charge is placed at each center. The distance between two centers varies from 2 to $5 \AA$.

from other methods can also be used in the present MIB technique. Before the MIB method is applied to actual biomolecules it is instructive to examine how the MIB method with such an approximate molecular surface description compares to traditional PB solvers for the simple diatomic test case shown in Figure 3.

The resulting electrostatic solvation energies for atomic distances $D=2,3,4,5 \AA$ are plotted in Figure 4. The electrostatic potential computed with the MIB method at a mesh size of $h=$ $0.05 \AA$ is chosen as the reference value in each case as the grid spacing $h$ is varied from 0.5 to $0.05 \AA$. The results illustrate that the solvation energy computed with the MIB methods is practically converged already at a grid spacing of $0.5 \AA$. The solvation energies from the standard finite difference solver deviate by about 4-6 kcal $/ \mathrm{mol}$ at a mesh size of $0.5 \AA$ but slowly converge to values close to the reference when a grid spacing of $0.05 \AA$ is reached. The convergence to the same answer can also be appreciated from the errors of surface potential with respect to the potential obtained with the MIB method at a $0.05 \AA$ grid spacing, shown in Figure 5. At $0.5 \AA$ resolution, the maximum and averaged relative errors of the surface potential with PBEQ are above $80 \%$ and $20 \%$, respectively. The errors decrease to about $60 \%$ and $10 \%$ respectively when a mesh of $0.1 \AA$ is used. All errors shown in Figure 5 are of the same magnitude as in the analytical test cases, suggesting that the approximate MSMS surface implemented with the MIB method does not introduce significant additional errors in electrostatic calculations of biomolecules.

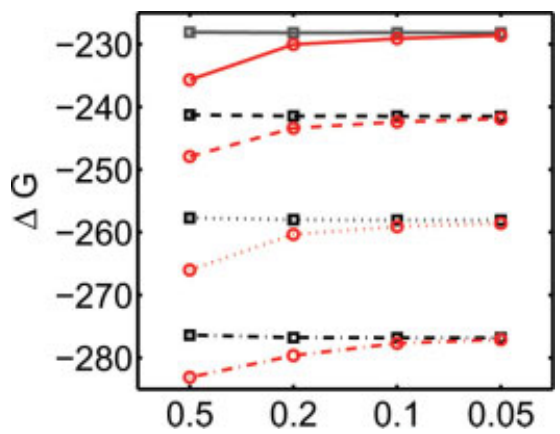

Figure 4. Convergence of computed electrostatic solvation energies $\Delta G$ in $\mathrm{kcal} / \mathrm{mol} v s$. mesh size $h$ in $\AA$. The results of the MIB and the PBEQ methods are marked with squares and circles, respectively. The results for $D=2,3,4,5$ are plotted as solid lines, dashed line, dotted lines, and dash-dotted lines, respectively. [Color figure can be viewed in the online issue, which is available at www.interscience.wiley.com.]
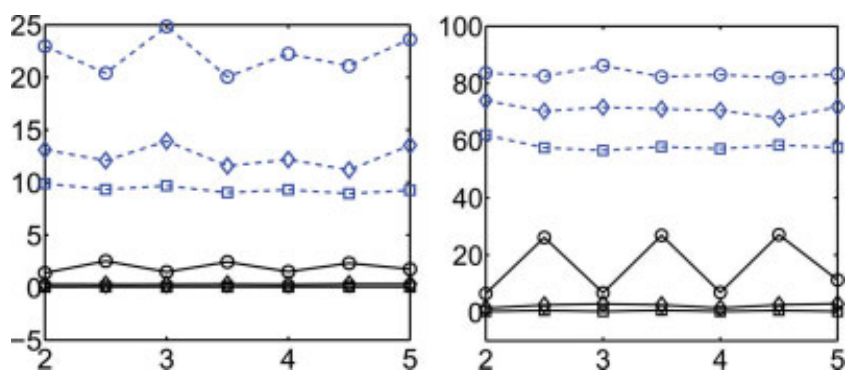

Figure 5. Maximum relative error $E_{2}$ (left) and average relative error $E_{3}$ (right) in the surface potential of two positively charged spheres. The errors with MIB and PBEQ methods are plotted in black and blue, respectively, $v s$. the distance between the centers of the two spheres in Å. Circles: $h=0.5$; diamonds: $h=0.2$; squares: $h=0.1$. [Color figure can be viewed in the online issue, which is available at www. interscience.wiley.com.]

\section{Biomolecular Electrostatic Solvation Free Energies}

After the preliminary tests, the MIB method was applied to the calculation of biomolecular solvation energies. Twenty-four proteins were selected from a large test set used in a previous study ${ }^{31}$ based on size and structural diversity (see Fig. 6). For all structures hydrogen atoms were added to obtain full all-atom models. Partial charges at atomic sites and atomic van der Waals radii defining the dielectric boundary were taken from the CHARMM22 force field..$^{52}$ Electrostatic solvation energies were calculated with the MIB method as well as PBEQ and APBS. Based on the results from the previous sections, it is expected that even a mesh size $h$ of $0.5 \AA$ is sufficient to obtain converged or nearly converged electrostatic solvation energies. Finer grid spacings were used with the PBEQ and APBS solvers. The results are shown in Figure 6. It is apparent that the solvation energies obtained with PBEQ and APBS differ significantly from the MIB results, but the difference is reduced for both PBEQ and APBS as finer mesh sizes are applied. Furthermore, the differences between the PBEQ/APBS and MIB results appear to increase as a function of the size of the protein. Note that the proteins are ordered with respect to their radius of gyration in all of the plots in Figure 6. These results suggest that the MIB method indeed provides converged, or at least more converged, estimates of the true electrostatic solvation energies. However, it is also found that at a very small grid spacing of $0.15 \AA$, solutions with PBEQ solutions slightly overshoot those found with MIB for three small proteins (PDB IDs: 1ajj, 1uxc and 1r69) suggesting that either convergence is also reached with PBEQ or the MIB results at a grid spacing of $0.5 \AA$ are not fully converged yet. However, for proteins with larger radii of gyration, the solvation energies computed with PBEQ at a grid spacing of $0.15 \AA$ are still much lower than those computed with the MIB solver. For protein 1 svr with 94 residues, for example, the deviation in the solvation energy is $-23 \mathrm{kcal} / \mathrm{mol}$. The results with APBS are similar albeit slightly larger than with PBEQ at the chosen grid resolutions.

Figure 6 also shows a comparison of the computational time needed to solve the PB equation with different methods which is of great practical importance. It can be seen that the computational cost of the MIB is very similar to the PBEQ solver at a grid spacing 
of $0.25 \AA$ and much less than the cost with APBS or PBEQ at the finer mesh sizes. At present, the MIB method is computationally more expensive than standard finite difference methods if the same grid spacing is compared, but the ability to obtain accurate solutions of the PB equation with the interface method at relatively coarse mesh sizes provides significant savings of computation time in calculating accurate electrostatic potentials and solvation energies for biomolecules. A drawback of the current implementation of the MIB method is a relatively large memory consumption due to the matrix structure and the algebraic solver that prohibits the application of finer mesh sizes for large molecules or the application of a $0.5 \AA$ grid to very large biomolecular complexes. Further algorithmic optimizations are undertaken to address these problems.

\section{Electrostatic Surface Potential}

Finally, surface electrostatic potentials were calculated with PBEQ and MIB for the RNA binding domain of E. coli $\rho$ factor (PBD ID: 1a63), which has 130 residues and 2069 atoms. Similar calculations are frequently carried out to understand the potential for protein-protein and protein-ligand interactions based on the dominant electrostatic contributions to intermolecular energetics.
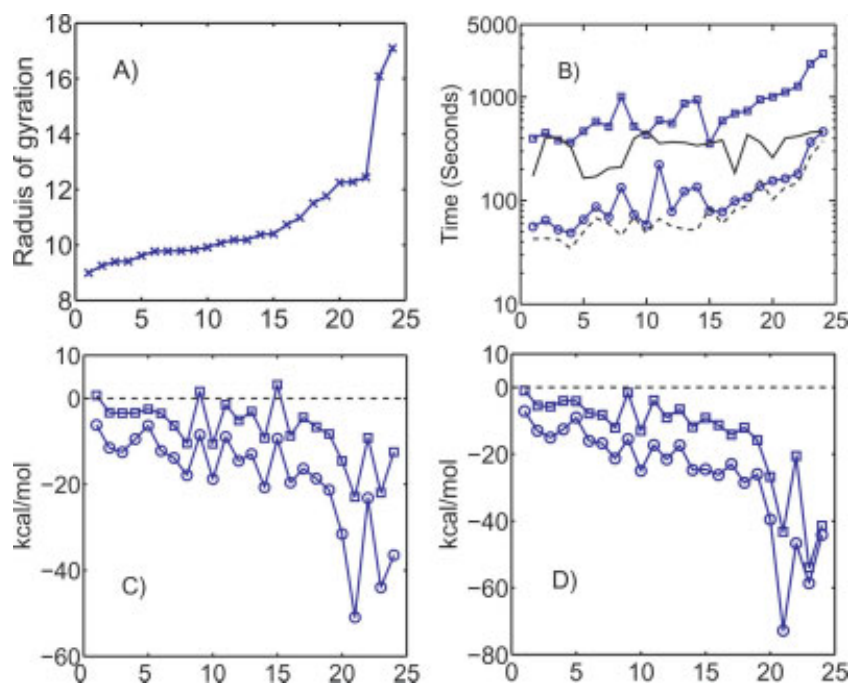

Figure 6. Computational results for 24 proteins. These proteins are ordered with increasing radii of gyration in each chart: 1ajj, 2pde, 1vii, 2erl, 1cbn, 1bor, 1bbl, 1fca, 1uxc, 1sh1, 1mbg, 1ptq, 1vjw, 1fxd, 1r69, 1hpt, 1bpi, 451c, 1a2s, 1frd, 1svr, 1neq, 1a63 and 1a7m. A: Radius of gyration in $\AA$. B: Computational time in seconds for different solvers at different grid spacing. Square: PBEQ at $0.15 \AA$ grid spacing; circle: PBEQ at $0.25 \AA$ grid spacing; solid line: APBS at about $0.2 \AA$ grid spacing; dashed line: MIB at $0.5 \AA$ grid spacing. C: Difference between computed electrostatic solvation energies. Square: between PBEQ solution at $0.15 \AA$ and MIB solution at $0.5 \AA$; circle: between PBEQ solution at $0.25 \AA$ and MIB solution at $0.5 \AA$. D: Difference between computed electrostatic solvation energies. Square: between APBS solution at about $0.2 \AA$ and MIB solution at $0.5 \AA$; circle: between APBS solution at about $0.4 \AA$ and MIB solution at $0.5 \AA$. [Color figure can be viewed in the online issue, which is available at www.interscience. wiley.com.]

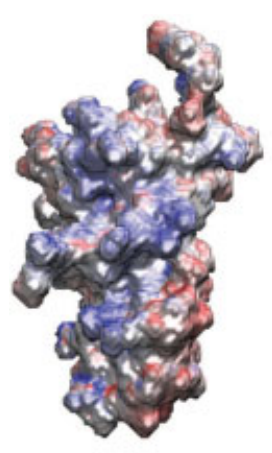

PBEQ

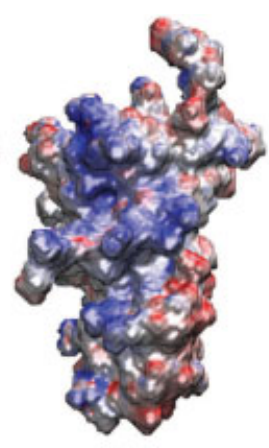

MIB

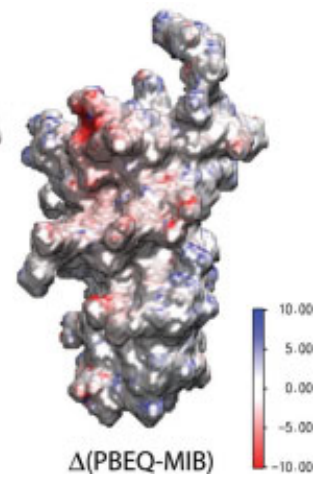

Figure 7. Electrostatic potentials of the RNA binding domain of $E$. coli $\rho$ factor (PBD ID: 1a63) projected onto its molecular surface. Left: Results from PBEQ (0.5 ̊ grid spacing); center: results from MIB (0.5 ̊ grid spacing); right: difference between PBEQ and MIB surface potentials. Colors are chosen according to the magnitude of the potential as indicated with the color bar in units of $\mathrm{kcal} / \mathrm{mol} / \mathrm{e}$.

Figure 7 shows the electrostatic potential projected at the molecular surface from the PBEQ and MIB methods as well as a difference map. It can be seen that the overall potential distribution is qualitatively very similar, but significant differences can be discerned. For example, part of the positively charged, RNA interacting region shown in the top part of the surface exhibits a significantly more positive surface potential with the MIB method than in the PBEQ solution. Such discrepancies of about $5 \mathrm{kcal} / \mathrm{mol} / \mathrm{e}$ would have consequences in a quantitative analysis of protein-RNA interactions.

\section{Discussion and Conclusions}

This work reports first applications of a novel Poisson-Boltzmann solver for calculating electrostatic potentials of biomolecules. The new method improves conventional finite difference methods by matched interface and boundary (MIB) method so that flux continuity of the electrostatic potential at dielectric interfaces is rigorously enforced. From a mathematical standpoint this greatly improves convergence properties as a function of mesh size. As a consequence, the practically achievable accuracy in solutions of the PB equation for typical biomolecular systems is dramatically improved.

Results for simple test cases where analytical answers are available for reference indicate that the MIB method is able to obtain essentially fully converged results with a relatively coarse mesh size of $0.5 \AA$. Conventional PB solvers can only come close to a similar level of accuracy and only when extremely fine and computationally costly meshes are employed. The application of the MIB method to small and medium-size biomolecules is more difficult to evaluate because an analytical exact answer is lacking for comparison. However, convergence of solvation energies from standard PB solvers toward the MIB results upon decreasing mesh sizes suggests that the MIB results are converged or nearly converged. In that case, the MIB results can serve as a reference for evaluating other PB solvers and more approximate methods such as the Generalized Born formalism. ${ }^{7}$ To our knowledge reference electrostatic 
potentials and solvation energies for biomolecules that are close to the theoretically exact solution are otherwise not available. If the MIB results are used as reference values, the established methods such as PBEQ and APBS would in fact exhibit significant deviations from fully converged solutions even at the finest grid spacings that can be computationally afforded with current hardware. Total electrostatic solvation energies may differ on the order of up to tens of kilocalories per mole depending on the size of the molecule; however, more concerning are the relatively large errors in the electrostatic potentials. While these errors may cancel in part when solvation energies are calculated according to eq. (19), detailed analysis of electrostatic potentials on protein surfaces may suffer to a larger extent both in quantitative and qualitative ways as illustrated for the example shown in Figure 7.

The MIB method is computationally more expensive than a standard finite difference solver if the same grid spacing is used with both methods. However, the extra computational cost is offset easily by only requiring relatively coarse grid spacings to obtain highly accurate solutions. It might be expected that even coarser grid spacings may still lead to acceptable accuracy on the order of what standard finite difference solvers can achieve with intermediate mesh sizes but at much reduced cost. However, the coarseness of the mesh is related to how close charges can approach the dielectric surface before significant errors are introduced in the MIB method. In the case of a $0.5 \AA$ grid spacing deviations become noticeable when charges come closer than about $1 \AA$ or two grid points. For biomolecular models based on classical force fields a $0.5 \AA$ grid spacing is therefore at the limit of what is acceptable for obtaining highly accurate solutions because atomic point charges are typically between 1 and $2 \AA$ away from the dielectric interface.

The main focus in this study has been the application of PB theory to biomolecules with a discontinuous dielectric boundary at the molecular surface. Although a sharp molecular surface is generally believed to be a good approximation of biomolecule-solvent interactions in the context of continuum models. ${ }^{12}$ Softer boundaries have also been used, usually to address numerical issues when calculating gradients for molecular dynamics simulations. ${ }^{44}$ However, it remains unclear whether such surfaces provide a more or less realistic description of biomolecule-environment interactions. ${ }^{31}$ While the MIB method is particularly well suited for obtaining electrostatic potentials in systems with sharp dielectric interfaces, its advantage over conventional finite difference solvers is expected to diminish with increasing smoothness of the interface.

The main achievement of the MIB method in the application to electrostatic calculations of biomolecular systems is the possibility to establish, for the first time, accurate reference points for the comparison and evaluation of other widely used dielectric continuum implicit solvent methods. Further studies based on the results from the MIB method are expected to distinguish more clearly physical limitations of continuum dielectric models from numerical inaccuracies in PB solvers and approximate formalisms in the continued development and application of such models to biomolecules.

\section{Acknowledgments}

Y. C. Zhou thanks Seiichiro Tanizaki for discussions and help with CHARMM calculations.

\section{Appendix}

Here we present the detailed expressions for twelve possible ways of eliminating two elements from a set of six partial derivatives.

1. Eliminating $\phi_{y}^{-}$and $\phi_{z}^{-}$from eqs. (7)-(9) to get

$$
\begin{aligned}
& {\left[\epsilon \phi_{\xi}\right]-\epsilon^{-} \tan \psi\left[\phi_{\zeta}\right]+C_{\eta}\left[\phi_{\eta}\right]} \\
& \quad=C_{x}^{+} \phi_{x}^{+}+C_{x}^{-} \phi_{x}^{-}+C_{y}^{+} \phi_{y}^{+}+C_{z}^{+} \phi_{z}^{+}
\end{aligned}
$$

where

$$
\begin{aligned}
C_{\eta}= & -\epsilon^{-} \tan \theta / \cos \psi \\
C_{x}^{+}= & \epsilon^{+} \cos \psi \cos \theta+\epsilon^{-} \tan \psi \sin \psi \cos \theta \\
& +\epsilon^{-} \sin \theta \tan \theta / \cos \psi \\
C_{x}^{-}= & -\epsilon^{-} /(\cos \psi \cos \theta) \\
C_{y}^{+}= & \left(\epsilon^{+}-\epsilon^{-}\right) \cos \psi \sin \theta \\
C_{z}^{+}= & \left(\epsilon^{+}-\epsilon^{-}\right) \sin \psi .
\end{aligned}
$$

2. Eliminating $\phi_{y}^{+}$and $\phi_{z}^{-}$from eqs. (7)-(9) to get

$$
\begin{aligned}
{\left[\epsilon \phi_{\xi}\right]+C_{\eta}\left[\phi_{\eta}\right] } & -\epsilon^{-} \tan \psi\left[\phi_{\zeta}\right] \\
& =C_{x}^{+} \phi_{x}^{+}+C_{x}^{-} \phi_{x}^{-}+C_{y}^{+} \phi_{y}^{+}+C_{z}^{-} \phi_{z}^{-}
\end{aligned}
$$

where

$$
\begin{aligned}
C_{\eta}= & -\epsilon^{+} \cos \psi \tan \theta-\epsilon^{-} \tan \psi \sin \psi \tan \theta \\
C_{x}^{+}= & \epsilon^{+} \cos \psi / \cos \theta+\epsilon^{-} \tan \psi \sin \psi / \cos \theta \\
C_{x}^{-}= & -\epsilon^{+} \cos \psi \tan \theta \sin \theta \\
& -\epsilon^{-}(\cos \psi \cos \theta \tan \psi \sin \psi / \cos \theta) \\
C_{y}^{-}= & \left(\epsilon^{+}-\epsilon^{-}\right) \cos \psi \sin \theta \\
C_{z}^{+}= & \left(\epsilon^{+}-\epsilon^{-}\right) \sin \psi .
\end{aligned}
$$

3. Eliminating $\phi_{y}^{-}$and $\phi_{z}^{+}$from eqs. (7)-(9) to get

$$
\begin{aligned}
{\left[\epsilon \phi_{\xi}\right]+C_{\eta}\left[\phi_{\eta}\right] } & -\epsilon^{-} \tan \psi\left[\phi_{\zeta}\right] \\
& =C_{x}^{+} \phi_{x}^{+}+C_{x}^{-} \phi_{x}^{-}+C_{y}^{+} \phi_{y}^{+}+C_{z}^{-} \phi_{z}^{-}
\end{aligned}
$$

where

$$
\begin{aligned}
C_{\eta}= & -\epsilon^{-} \cos \psi \tan \theta-\epsilon^{+} \tan \psi \sin \psi \tan \theta \\
C_{x}^{+}= & \epsilon^{-} \tan \theta \cos \psi \sin \theta \\
& +\epsilon^{+}(\cos \psi \cos \theta+\tan \psi \sin \psi / \cos \theta) \\
C_{x}^{-}= & -\epsilon^{-} \cos \psi / \cos \theta-\epsilon^{+} \sin \psi \tan \psi / \cos \theta \\
C_{y}^{+}= & \left(\epsilon^{+}-\epsilon^{-}\right) \cos \psi \sin \theta \\
C_{z}^{-}= & \left(\epsilon^{+}-\epsilon^{-}\right) \sin \psi .
\end{aligned}
$$

4. Eliminating $\phi_{y}^{+}$and $\phi_{z}^{+}$from eqs. (7)-(9) to get

$$
\begin{aligned}
{\left[\epsilon \phi_{\xi}\right]+C_{\eta}\left[\phi_{\eta}\right] } & -\epsilon^{+} \tan \psi\left[\phi_{\zeta}\right] \\
& =C_{x}^{+} \phi_{x}^{+}+C_{x}^{-} \phi_{x}^{-}+C_{y}^{-} \phi_{y}^{-}+C_{z}^{-} \phi_{z}^{-}
\end{aligned}
$$


where

$$
\begin{aligned}
C_{\eta}= & -\epsilon^{+} \tan \theta / \cos \psi \\
C_{x}^{+}= & \epsilon^{+} /(\cos \psi \cos \theta) \\
C_{x}^{-}= & -\epsilon^{-} \cos \psi \cos \theta \\
& -\epsilon^{+}(\tan \psi \sin \psi \cos \theta+\tan \theta \sin \theta / \cos \psi) \\
C_{y}^{-}= & \left(\epsilon^{+}-\epsilon^{-}\right) \cos \psi \sin \theta \\
C_{z}^{-}= & \left(\epsilon^{+}-\epsilon^{-}\right) \sin \psi .
\end{aligned}
$$

5. Eliminating $\phi_{x}^{-}$and $\phi_{z}^{-}$from eqs. (7)-(9) to get

$$
\begin{aligned}
{\left[\epsilon \phi_{\xi}\right]+C_{\eta}\left[\phi_{\eta}\right] } & -\epsilon^{-} \tan \psi\left[\phi_{\zeta}\right] \\
& =C_{y}^{+} \phi_{y}^{+}+C_{y}^{-} \phi_{y}^{-}+C_{x}^{+} \phi_{x}^{+}+C_{z}^{+} \phi_{z}^{+}
\end{aligned}
$$

where

$$
\begin{aligned}
C_{\eta}= & \epsilon^{-} \cot \theta / \cos \psi \\
C_{y}^{+}= & \epsilon^{+} \cos \psi \sin \theta \\
& +\epsilon^{-}(\cot \theta \cos \theta / \cos \psi+\tan \psi \sin \psi \sin \theta) \\
C_{y}^{-}= & -\epsilon^{-} /(\cos \psi \sin \theta) \\
C_{x}^{+}= & \left(\epsilon^{+}-\epsilon^{-}\right) \cos \psi \cos \theta \\
C_{z}^{+}= & \left(\epsilon^{+}-\epsilon^{-}\right) \sin \psi .
\end{aligned}
$$

6. Eliminating $\phi_{x}^{-}$and $\phi_{z}^{+}$from eqs. (7)-(9) to get

$$
\begin{aligned}
{\left[\epsilon \phi_{\xi}\right]+C_{\eta}\left[\phi_{\eta}\right] } & -\epsilon^{+} \tan \psi\left[\phi_{\zeta}\right] \\
& =C_{y}^{+} \phi_{y}^{+}+C_{y}^{-} \phi_{y}^{-}+C_{x}^{+} \phi_{x}^{+}+C_{z}^{-} \phi_{z}^{-}
\end{aligned}
$$

where

$$
\begin{aligned}
C_{\eta}= & \epsilon^{-} \cos \psi \cot \theta+\epsilon^{+} \tan \psi \sin \psi \cot \theta \\
C_{y}^{+}= & \epsilon^{+} \cos \psi \sin \theta+\epsilon^{-} \cos \psi \cos \theta \cot \theta \\
& +\epsilon^{+} \tan \psi \sin \psi / \sin \theta \\
C_{y}^{-}= & -\left(\epsilon^{-} \cos \psi+\epsilon^{+} \tan \psi \sin \psi\right) / \sin \theta \\
C_{x}^{+}= & \left(\epsilon^{+}-\epsilon^{-}\right) \cos \psi \cos \theta \\
C_{z}^{-}= & \left(\epsilon^{+}-\epsilon^{-}\right) \sin \psi .
\end{aligned}
$$

7. Eliminating $\phi_{x}^{+}$and $\phi_{z}^{-}$from eqs. (7)-(9) to get

$$
\begin{aligned}
{\left[\epsilon \phi_{\xi}\right]+C_{\eta}\left[\phi_{\eta}\right] } & -\epsilon^{-} \tan \psi\left[\phi_{\zeta}\right] \\
& =C_{y}^{+} \phi_{y}^{+}+C_{y}^{-} \phi_{y}^{-}+C_{x}^{-} \phi_{x}^{-}+C_{z}^{+} \phi_{z}^{+}
\end{aligned}
$$

where

$$
\begin{aligned}
C_{\eta}= & \epsilon^{+} \cos \psi \cot \theta+\epsilon^{-} \tan \psi \sin \psi \cot \theta \\
C_{y}^{+}= & \left(\epsilon^{+} \cos \psi+\epsilon^{-} \tan \psi \sin \psi\right) / \sin \theta \\
C_{y}^{-}= & -\epsilon^{-} \cos \psi \sin \theta-\epsilon^{+} \cos \psi \cos \theta \cot \theta \\
& -\epsilon^{-} \tan \psi \sin \psi / \sin \theta \\
C_{x}^{-}= & \left(\epsilon^{+}-\epsilon^{-}\right) \cos \psi \cos \theta \\
C_{z}^{+}= & \left(\epsilon^{+}-\epsilon^{-}\right) \sin \psi .
\end{aligned}
$$

8. Eliminating $\phi_{x}^{+}$and $\phi_{z}^{+}$from eqs. (7)-(9) to get

$$
\begin{aligned}
{\left[\epsilon \phi_{\xi}\right]+C_{\eta}\left[\phi_{\eta}\right] } & -\epsilon^{+} \tan \psi\left[\phi_{\zeta}\right] \\
& =C_{y}^{+} \phi_{y}^{+}+C_{y}^{-} \phi_{y}^{-}+C_{x}^{-} \phi_{x}^{-}+C_{z}^{-} \phi_{z}^{-}
\end{aligned}
$$

where

$$
\begin{aligned}
C_{\eta}= & \epsilon^{+} \cot \theta / \cos \psi \\
C_{y}^{+}= & \epsilon^{+} /(\cos \psi \sin \theta) \\
C_{y}^{-}= & -\epsilon^{-} \cos \psi \sin \theta-\epsilon^{+} \cos \psi \cos \theta \cot \theta \\
& -\epsilon^{+} \tan \psi \sin \psi / \sin \theta \\
C_{x}^{-}= & \left(\epsilon^{+}-\epsilon^{-}\right) \cos \psi \cos \theta \\
C_{z}^{-}= & \left(\epsilon^{+}-\epsilon^{-}\right) \sin \psi .
\end{aligned}
$$

9. Eliminating $\phi_{x}^{-}$and $\phi_{y}^{-}$from eqs. (7)-(9) to get

$$
\begin{aligned}
{\left[\epsilon \phi_{\xi}\right]+C_{\eta}\left[\phi_{\eta}\right] } & +C_{\zeta}\left[\phi_{\zeta}\right] \\
& =C_{z}^{+} \phi_{z}^{+}+C_{z}^{-} \phi_{z}^{-}+C_{x}^{+} \phi_{x}^{+}+C_{y}^{+} \phi_{y}^{+}
\end{aligned}
$$

where

$$
\begin{aligned}
& C_{\eta}=0 \\
& C_{\zeta}=\epsilon^{-} \cot \psi \\
& C_{z}^{+}=\epsilon^{+} \sin \psi+\epsilon^{-} \cos \psi \cot \psi \\
& C_{z}^{-}=-\epsilon^{-} / \sin \psi \\
& C_{x}^{+}=\left(\epsilon^{+}-\epsilon^{-}\right) \cos \psi \cos \theta \\
& C_{y}^{+}=\left(\epsilon^{+}-\epsilon^{-}\right) \cos \psi \sin \theta .
\end{aligned}
$$

10. Eliminating $\phi_{x}^{-}$and $\phi_{y}^{+}$from eqs. (7)-(9) to get

$$
\begin{aligned}
{\left[\epsilon \phi_{\xi}\right]+C_{\eta}\left[\phi_{\eta}\right] } & +C_{\zeta}\left[\phi_{\zeta}\right] \\
& =C_{z}^{+} \phi_{z}^{+}+C_{z}^{-} \phi_{z}^{-}+C_{x}^{+} \phi_{x}^{+}+C_{y}^{-} \phi_{y}^{-}
\end{aligned}
$$

where

$$
\begin{aligned}
C_{\eta}= & -\left(\epsilon^{+}-\epsilon^{-}\right) \cos \psi \sin \theta \cos \theta \\
C_{\zeta}= & \epsilon^{+} \cot \psi \sin ^{2} \theta+\epsilon^{-} \cot \psi \cos ^{2} \theta \\
C_{z}^{+}= & \epsilon^{+} \sin \psi+\epsilon^{+} \cos \psi \cot \psi \sin ^{2} \theta \\
& +\epsilon^{-} \cos \psi \cot \psi \cos ^{2} \theta \\
C_{z}^{-}= & -\epsilon^{-} \sin \psi-\epsilon^{+} \cos \psi \cot \psi \sin ^{2} \theta \\
& -\epsilon^{-} \cos \psi \cot \psi \cos ^{2} \theta \\
C_{x}^{+}= & \left(\epsilon^{+}-\epsilon^{-}\right) \cos \psi \cos \theta \\
C_{y}^{-}= & \left(\epsilon^{+}-\epsilon^{-}\right) \cos \psi \sin \theta
\end{aligned}
$$

11. Eliminating $\phi_{x}^{+}$and $\phi_{y}^{-}$from eqs. (7)-(9) to get

$$
\begin{aligned}
{\left[\epsilon \phi_{\xi}\right]+C_{\eta}\left[\phi_{\eta}\right] } & +C_{\zeta}\left[\phi_{\zeta}\right] \\
& =C_{z}^{+} \phi_{z}^{+}+C_{z}^{-} \phi_{z}^{-}+C_{x}^{-} \phi_{x}^{-}+C_{y}^{+} \phi_{y}^{+}
\end{aligned}
$$


where

$$
\begin{aligned}
C_{\eta}= & \left(\epsilon^{+}-\epsilon^{-}\right) \cos \psi \sin \theta \cos \theta \\
C_{\zeta}= & \epsilon^{+} \cot \psi \cos ^{2} \theta+\epsilon^{-} \cot \psi \sin ^{2} \theta \\
C_{z}^{+}= & \epsilon^{+} \sin \psi+\epsilon^{+} \cos \psi \cot \psi \cos ^{2} \theta \\
& +\epsilon^{-} \cos \psi \cot \psi \sin ^{2} \theta \\
C_{z}^{-}= & -\epsilon^{-} \sin \psi-\epsilon^{+} \cos \psi \cot \psi \cos ^{2} \theta \\
& -\epsilon^{-} \cos \psi \cot \psi \sin ^{2} \theta \\
C_{x}^{-}= & \left(\epsilon^{+}-\epsilon^{-}\right) \cos \psi \cos \theta \\
C_{y}^{+}= & \left(\epsilon^{+}-\epsilon^{-}\right) \cos \psi \sin \theta .
\end{aligned}
$$

12. Eliminating $\phi_{x}^{+}$and $\phi_{y}^{+}$from eqs. (7)-(9) to get

$$
\begin{aligned}
{\left[\epsilon \phi_{\xi}\right]+C_{\eta}\left[\phi_{\eta}\right] } & +C_{\zeta}\left[\phi_{\zeta}\right] \\
& =C_{z}^{+} \phi_{z}^{+}+C_{z}^{-} \phi_{z}^{-}+C_{x}^{-} \phi_{x}^{-}+C_{y}^{-} \phi_{y}^{-}
\end{aligned}
$$

where

$$
\begin{aligned}
C_{\eta} & =0 \\
C_{\zeta} & =\epsilon^{+} \cot \psi \\
C_{z}^{+} & =\epsilon^{+} / \sin \psi \\
C_{z}^{-} & =-\epsilon^{-} \sin \psi-\epsilon^{+} \cos \psi \cot \psi \\
C_{x}^{-} & =\left(\epsilon^{+}-\epsilon^{-}\right) \cos \psi \cos \theta \\
C_{y}^{-} & =\left(\epsilon^{+}-\epsilon^{-}\right) \cos \psi \sin \theta .
\end{aligned}
$$

\section{References}

1. Israelchvili, J. N. Intermolecular and Surface Forces; Academic Press: London, 1900.

2. Hunter, R. J. Foundations of Colloid Science; Oxford University Press: New York, 1989.

3. Andelman, D. Electrostatic Properties of Membranes: The PoissonBoltzmann Theory; 1995, Handbook of Biological Physics, Vol. 1, 603.

4. Scott, R.; Boland, M.; Rogale, K.; Fernández, A. J Phys A: Math Gen 2004, 37, 9791.

5. Khohlova, S. S.; Mikhailova, V. A.; Ivanov, A. I. J Chem Phys 2006, $124,114507$.

6. Baker, N. A. Curr Opin Struct Biol 2005, 15, 137.

7. Feig, M.; Brooks, C. L., III. Curr Opin Struct Biol 2004, 14, 217.

8. Cortis, C. M.; Friesner, R. A. J Comput Chem 1997, 18, 1591.

9. Forsten, K. E.; Kozack, R. E.; Lauffenburger, D. A.; Subramaniam, S. J Phys Chem 1994, 98, 5580.

10. Honig, B.; Nicholls, A. Science 1995, 268, 1144.

11. Roux, B.; Simonson, T. Biophys Chem 1999, 78, 1.

12. Sharp, K. A.; Honig, B. Annu Rev Biophys Biophys Chem 1990, 19, 301.

13. Vorobjev, Y. N.; Scheraga, H. A. J Comput Chem 1997, 18, 569.

14. Zauhar, R. J.; Morgan, R. S. J Mol Biol 1985, 186, 815.
15. Fogolari, F.; Brigo, A.; Molinari, H. Biophys J 2003, 85, 159.

16. Kollman, P. A.; Massova, I.; Reyes, C.; Kuhn, B.; Huo, S.; Chong, L.; Lee, M.; Lee, T.; Duan, Y.; Wang, W.; Donini, O.; Cieplak, P.; Srinivasan, J.; Case, D. A.; Cheathem, T. E., III. Acc Chem Res 2000, 33, 889.

17. Swanson, J. M. J.; Henchman, R. H.; McCammon, J. A. Biophys J 2004, $86,67$.

18. Lu, Q.; Luo, R. J Chem Phys 2003, 119, 11035.

19. Prabhu, N. V.; Zhu, P.; Sharp, K. A. J Comput Chem 2004, 25, 2049.

20. Zhou, R.; Berne, B. J. Proc Natl Acad Sci 2002, 99, 12777.

21. Zagrovic, B.; Sorin, E.; Pande, V. J Mol Biol 2001, 313, 151.

22. Tanizaki, S.; Feig, M. J Phys Chem B 2006, 110, 548.

23. Gallicchio, E.; Levy, R. M. J Comp Chem 2004, 25, 479.

24. Jang, S.; Shin, S.; Pak, Y. J Am Chem Soc 2002, 124, 4976.

25. Gilson, M. K.; Honig, B. Proteins 1988, 4, 7.

26. Bashford, D. Front Biosci 2004, 9, 1082.

27. Luty, B. A.; Davis, M. E.; McCammon, J. A. J Comp Chem 1992, 13, 1114

28. Baker, N. A.; Sept, D.; Joseph, S.; Holst, M. J.; McCammon, J. A. Proc Natl Acad Sci 2001, 98, 10037.

29. Boschitsch, A. H.; Fenley, M. O.; Zhou, H. X. J Phys Chem B 2005, $106,214102$.

30. Lu, B. Z.; Zhang, D. Q.; McCammon, J. A. J Chem Phys 2002, 122, 2741.

31. Feig, M.; Onufriev, A.; Lee, M. S.; Im, W.; Case, D. A.; Brooks, C. L. III. J Comp Chem 2004, 25, 265.

32. Pettitt, B. M.; Valdeavella, C. V. In Simulation and Theory of Electrostatic Interactions in Solution; Pratt, L. R.; Hummer, G., Eds.; AIP Press: New York, 1999; pp. 411.

33. Connolly, M. L. J Appl Cryst 1983, 16, 548.

34. Babuška, I. Computing 1970, 5, 207.

35. Gilson, M. K.; Sharp, K. A.; Honig, B. J Comp Chem 1987, 9, 327.

36. Holst, M. Ph.D. thesis, Numerical Computing Group, University of Illinois at Urbana-Champaign, 1993; pp 11.

37. Peskin, C. S. J Comput Phys 1977, 25, 220.

38. LeVeque, R. J.; Li, Z. L. SIAM J Numer Anal 1994, 31, 1019.

39. Fedkiw, R. P.; Aslam, T.; Merriman, B.; Osher, S. J Comput Phys 1999, $152,457$.

40. Zhao, S.; Wei, G. W. J Comput Phys 2004, 200, 60.

41. Zhou, Y. C.; Zhao, S.; Feig, M.; Wei, G. W. J Comput Phys 2006, 213,1 .

42. Zhou, Y. C.; Wei, G. W. J Comput Phys 2006, 219, 228.

43. Sining Yu, Zhou, Y. C.; Wei, G. W. J Comput Phys (in press).

44. Im, W.; Beglov, D.; Roux, B. Comp Phys Comm 1998, 111, 59.

45. Brooks, B. R.; Bruccoleri, R. E.; Olafson, B. D.; States, D. J.; Swaminathan, S.; Karplus, M. J Comp Chem 1983, 4, 187.

46. Holst, M.; Baker, N.; Wang, F. J Comp Chem 2000, 21, 1319.

47. Baker, N.; Holst, M.; Wang, F. J Comp Chem 2000, 21, 1343.

48. Holst, M.; Saied, F. J Comput Chem 1993, 14, 105.

49. Holst, M.; Saied, F. J Comput Chem 1995, 16, 337.

50. Kirkwood, J. G. J Chem Phys 1934, 7, 351.

51. Sanner, M.; Olson, A.-J.; Spehner, J.-C. Biopolymers 1996, 38, 305.

52. MacKerell, Jr. A. D.; Bashford, D.; Bellott, M.; Dunbrack, J. D.; Evanseck, M. J.; Field, M. J.; Fischer, S.; Gao, J.; Guo, H.; Ha, S.; Joseph-McCarthy, D.; Kuchnir, L.; Kuczera, K.; Lau, F. T. K.; Mattos, C.; Michnick, S.; Ngo, T.; Nguyen, D. T.; Prodhom, B.; Reiher, W. E.; Roux, B.; Schlenkrich, M.; Smith, J. C.; Stote, R.; Straub, J.; Watanabe, M.; Wiorkiewicz-Kuczera, J.; Yin, D.; Karplus, M. J Phys Chem 1998, 102,3586 . 